\title{
Optimisation multi-objectifs à base de métamodèle pour des applications en mise en forme des métaux
}

\author{
Mohsen Ejday a et Lionel Fourment \\ Mines ParisTech, CEMEF - Centre de Mise en Forme des Matériaux, CNRS UMR 7635, BP 207, 1 rue Claude Daunesse, \\ 06904 Sophia Antipolis Cedex, France
}

Reçu le 8 juillet 2010, accepté le 12 juillet 2010

Résumé - Pour appliquer les algorithmes d'optimisation multi-objectifs à des problèmes de mise en forme des métaux très coûteux en temps de calcul, nous étudions le couplage de l'algorithme génétique NSGA-II proposé par Deb à un métamodèle inspiré de la méthode des différences finies sans maillage de Liszka et Orkisz. Nous soulignons l'importance d'améliorer itérativement le métamodèle au cours des itérations d'optimisation, et la possibilité de déterminer avec précision des fronts optimaux de Pareto des problèmes multi-objectifs étudiés en moins d'une centaine de calculs.

Mots clés : Optimisation multi-objectifs / différences finis sans maillage / NSGA-II / métamodèle / mise en forme des métaux

\begin{abstract}
Metamodel assisted multi-objective optimization for metal forming applications. To apply multi-objective optimization algorithms to highly time expensive metal forming applications, the coupling of the NSGA-II genetic algorithm proposed by Deb with metamodels based on the Meshless Finite Difference Method (MFDM) proposed by Liszka and Orkisz is investigated. The importance of iteratively improving the metamodel during the optimization iterations is highlighted, as well as the capability to accurately determine the Pareto optimal fronts of the studied problems within less than hundred calculations.
\end{abstract}

Key words: Multi-objective optimization / meshless finite difference method / NSGA-II / metal forming

\section{Introduction}

Pour les Problèmes d'Optimisation Multi-Objectifs (POMO), il est d'usage de ne pas rechercher une seule solution optimale, mais un ensemble de solutions optimales correspondant aux différents arbitrages possibles entre les fonctions optimisées. La représentation graphique de cet ensemble dans l'espace fonctionnel est nommée front optimal de Pareto [1]. Les Algorithmes Évolutionnaires MultiObjectifs (AEMO) apparaissent comme les techniques les plus générales et les plus efficaces pour la détermination de cet ensemble avec une bonne répartition de ses points, même pour des problèmes particulièrement difficiles [1-3]. Cependant, les AEMO nécessitent un très grand nombre d'évaluations des fonctions coût, ce qui rend ces stratégies inutilisables pour des problèmes gourmands en temps de calcul, comme cela est le cas en mise en forme des matériaux, le cadre de notre étude.

Ce nombre important d'évaluations peut être considérablement réduit en utilisant des approximations

\footnotetext{
a Auteur pour correspondance :

lionel.fourment@mines-paristech.fr
}

construites à partir d'un modèle approché ou métamodèle. Dans le cadre de ce travail, nous nous proposons d'utiliser un métamodèle inspiré des méthodes de différences finies sans maillage initialement proposé par Liszka et Orkisz [4], et de nous limiter à un nombre maximum d'évaluations fixé arbitrairement à 100. Le couplage de l'AEMO au Métamodèle (M-AEMO) peut être effectué de plusieurs manières, dont l'étude constitue le corps de cet article. Cet algorithme est ensuite évalué sur plusieurs fonctions analytiques tests, avant d'être appliqué à deux POMOs de forgeage 3D.

\section{Problème d'optimisation multi-objectifs}

Un Problème d'Optimisation Multi-Objectifs (POMO) est constitué de $M$ fonctions coût $\phi_{m}(X)$ à minimiser ou maximiser par rapport à $X$, le vecteur des $n$ variables de contrôle (ou paramètres) : $X=\left(x_{1}, x_{2}, \ldots, x_{n}\right)^{T}$. Dans la pratique, il doit souvent satisfaire un certain nombre de contraintes, explicites $\left(x_{i}^{I} \leq x_{i} \leq x_{i}^{s}, \forall i=1, n\right)$ ou implicites, d'égalité 


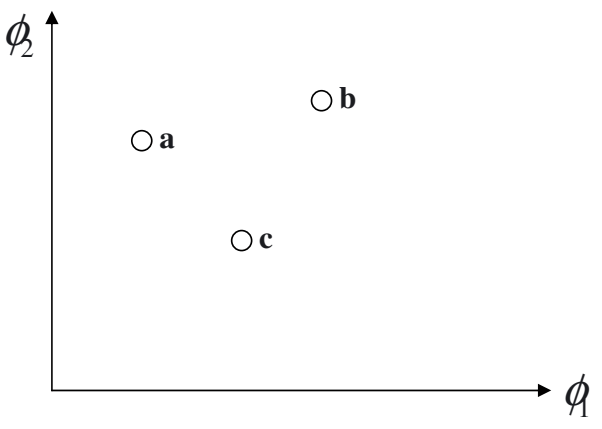

Fig. 1. Illustration du critère de dominance pour un problème de minimisation à deux fonctions coût : la solution (a) domine la solution (b) car elle est meilleure pour les deux fonctions coût. La solution (a) est indifférente de la solution (c) car chacune est meilleure pour une fonction coût.

$\left(h_{k}(X)=0, \forall k=1, k\right)$ ou d'inégalité $\left(g_{j}(X) \geq 0\right.$, $\forall j=1, J)$. L'ensemble des solutions vérifiant ces contraintes constitue l'espace de recherche (ou de décision) $S$. Le POMO peut donc s'écrire sous la forme générale :

$$
\begin{array}{r}
\text { Minimiser/Maximiser } \phi_{m}(X), \quad \forall m=1, M \\
\text { satisfaisant }\left\{\begin{array}{l}
g_{j}(X) \geq 0, \quad \forall j=1, J \\
h_{k}(X)=0, \quad \forall k=1, K \\
x_{i}^{I} \leq x_{i} \leq x_{i}^{s}, \quad \forall i=1, n
\end{array}\right.
\end{array}
$$

Pour déterminer l'ensemble des solutions optimales au sein de l'espace de décision, on utilise le critère de dominance $[1,5-10]$ qui est illustré par la figure 1 . Pour un problème de minimisation, une solution $X^{1}$ est dite dominer une solution $X^{2}$, si les deux conditions suivantes sont vraies :

1. $\forall j=1, \ldots, M, \phi_{j}\left(X^{1}\right) \leq \phi_{j}\left(X^{2}\right)$

2. $\exists j \operatorname{tq} \phi_{j}\left(X^{1}\right)<\phi_{j}\left(X^{2}\right)$ : on dit que $\phi_{j}\left(X^{1}\right)$ est meilleure que $\phi_{j}\left(X^{2}\right)$

Les solutions $X^{1}$ et $X^{2}$ sont dites indifférentes si et seulement si aucune d'elles ne domine l'autre. L'ensemble des solutions non-dominées de $S$ forme l'ensemble des solutions optimales; il est connu sous le nom d'ensemble optimal de Pareto et sa représentation graphique dans l'espace fonctionnel est nommée front optimal de Pareto. La résolution d'un POMO consiste à atteindre deux objectifs : (a) trouver un ensemble de solutions qui converge vers le front de Pareto et (b) que cet ensemble soit suffisamment riche ou bien distribué pour représenter au mieux l'ensemble du front de Pareto.

\section{Algorithme génétique élitiste de tri non-dominé (NSGA-II)}

L'algorithme génétique NSGA-II proposé par Deb $[1,5]$, et également présenté dans $[6,7]$, est souvent considéré comme l'un des algorithmes les plus efficaces pour trouver l'ensemble optimal de Pareto avec une excellente variété de solutions. Il repose sur les trois caractéristiques suivantes : (a) il utilise le principe de l'élitisme, (b) il favorise les solutions non dominées, et (c) il développe une variété explicite des solutions.

Le NSGA-II commence par la génération aléatoire d'une population initiale $P_{0}$ de $N$ individus parents. Ensuite, à chaque génération $t$, une population $Q_{t}$ de $N$ enfants est créée à partir de la population parent $P_{t}$, en utilisant les opérateurs génétiques habituels (sélection croisement - mutation). Les deux populations sont combinées pour former une nouvelle population $R_{t}$ de taille $2 N$. Le concept de dominance [1] permet de classer les individus de $R_{t}$ en plusieurs fronts de rangs différents. Le tri est effectué de la manière suivante : chaque individu de $R_{t}$ est comparé à tous les autres individus en se basant sur le critère de dominance. Les individus non dominés appartiennent au front de rang 1, le front optimal de Pareto. En éliminant, temporairement, ces individus de l'ensemble de recherche, l'algorithme est repris pour calculer le front de rang 2 , et ainsi de suite. La nouvelle population parent $P_{t+1}$ est alors construite à partir des $N$ individus appartenant aux fronts de rang les plus faibles. Pour le dernier front retenu, il y a en général plus de solutions possibles que de places restantes dans la nouvelle population parent $P_{t+1}$. Les individus sont alors triés selon leur distance d'encombrement sur le front $[1,8]$, et par ordre décroissant. Cette distance est une mesure de l'écart entre deux points successifs appartenant au front considéré. Ce choix permet de retenir la meilleure distribution des individus sur le front de rang le plus élevé.

Le principal inconvénient des algorithmes évolutionnaires est leur coût de calcul, très important en nombre d'évaluation des fonctions, et qui est généralement de plusieurs milliers. Pour surmonter cet obstacle, un modèle approché ou métamodèle est proposé.

\section{Métamodèle}

Dans la littérature, on rencontre plusieurs types de métamodèles : les métamodèles basés sur des interpolations gaussiennes (Krigeage) [9, 11-14], les techniques de surface de réponses $[10,14]$, et les réseaux de neurones artificiels [5]. Le métamodèle utilisé dans ce travail est basé sur la méthode de différences finies sans maillage de Liszka et Orkisz $[15,16]$, qui peut être considérée comme faisant partie de la grande famille des méthodes de type moindres carrés mobiles [10,17] ou encore plus généralement des méthodes d'interpolation sans maillage. Ce métamodèle repose sur une interpolation locale d'ordre deux, construite à partir de développements limités du second ordre. Considérons un point $i$ de l'espace paramétrique, où nous souhaitons calculer une approximation $\tilde{\phi}_{i}$ de $\phi_{i}=\phi\left(X_{i}\right)$, à partir de la connaissance de la valeur de $\phi_{j}$ aux $\lambda$ points maîtres $j$ du domaine. Pour cela, nous écrivons un développement limité à l'ordre deux de $\phi_{i}$ en $j$ :

$$
\begin{aligned}
\phi\left(X_{j}\right)= & \phi\left(X_{i}\right)+{ }^{t} \nabla \phi\left(X_{i}\right)\left(X_{j}-X_{i}\right)+\frac{1}{2}{ }^{t}\left(X_{j}-X_{i}\right) \\
& \times \nabla^{2} \phi\left(X_{i}\right)\left(X_{j}-X_{i}\right)+O\left(\left\|X_{j}-X_{i}\right\|^{3}\right)
\end{aligned}
$$


Notons :

$$
\left\{\begin{array}{l}
a=\phi\left(X_{i}\right)=\phi_{i}, \quad \text { et } x_{i j}=X_{j}-X_{i} \\
b_{k}=\left(\nabla \phi\left(X_{i}\right)\right)_{k}=\left(\nabla \phi_{i}\right)_{k}, \forall k=1, n \\
c_{k l}=\left(\nabla^{2} \phi\left(X_{i}\right)\right)_{k l}=\left(\nabla^{2} \phi_{i}\right)_{k l}, \forall k=1, n, \forall l=1, n
\end{array}\right.
$$

Le développement limité peut être réécrit sous la forme suivante :

$$
\begin{aligned}
\phi_{j}= & \phi_{j}\left(X_{j}\right)=a+\sum_{k=1}^{n} b_{k} x_{i j}^{k}+\frac{1}{2} \sum_{k, l=1}^{n} c_{k l} x_{i j}^{k} x_{i j}^{l} \\
& +O\left(\left\|x_{i j}\right\|^{3}\right)
\end{aligned}
$$

Nous pouvons alors calculer la solution $(a, b, c)$ qui minimise l'erreur quadratique moyenne $E(a, b, c)$ (Éq. (4)) de l'ensemble des développements limités (Éq. (3)) écrits pour les $\lambda$ points maîtres.

$$
\begin{aligned}
E(a, b, c)= & \frac{1}{2} \sum_{j=1}^{\lambda} \omega_{j}\left(a+\sum_{k=1}^{n} b_{k} x_{i j}^{k}\right. \\
& \left.+\frac{1}{2} \sum_{k, l=1}^{n} c_{k l} x_{i j}^{k} x_{i j}^{l}-\phi_{j}\right)^{2}
\end{aligned}
$$

où la fonction poids $\omega_{j}=\left\|x_{i j}\right\|^{-6}$ découle de l'ordre d'approximation du développement limité (voir Éq. (3)) ; le terme entre parenthèses (l'ordre de l'erreur) est donc proportionnel à $\left\|x_{i j}\right\|^{3}$. Si $\left\|x_{i j}\right\|$ est proche de zéro, on considère que le point $i$ est confondu avec le point maître $j$ et donc que $\tilde{\phi}_{i}=\phi_{j}$. Ainsi, cette méthode ne nécessite pas de paramétrage particulier, contrairement par exemple à la méthode des moindres carrées mobiles $[10,17]$ qui utilise un rayon d'influence dans la définition de ses fonctions fenêtre, de manière à éliminer l'influence des points éloignés. La détermination de ce rayon peut s'avérer délicate dans les cas les plus généraux, alors qu'ici tous les points maîtres sont utilisés, avec une influence toutefois fortement décroissante avec la distance.

La minimisation de l'erreur quadratique moyenne (Éq. (4)) conduit à résoudre un système linéaire (Éq. (5)) de $1+n+n \frac{(n+1)}{2}$ équations à $1+n+n \frac{(n+1)}{2}$ inconnues :

$$
A X=B
$$

avec :

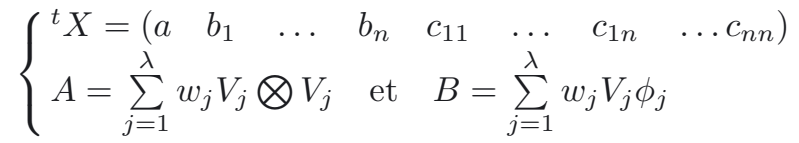

où :

$$
\begin{aligned}
{ }^{t} V_{j}= & \left(1 x_{i j}^{1} \ldots x_{i j}^{n} 1 / 2\left(x_{i j}^{1}\right)^{2} x_{i j}^{1} x_{i j}^{2} \ldots x_{i j}^{1} x_{i j}^{n}\right. \\
& \left.1 / 2\left(x_{i j}^{2}\right)^{2} \ldots 1 / 2\left(x_{i j}^{n}\right)^{2}\right)
\end{aligned}
$$

La résolution de l'équation (5) permet de calculer la solution $n(a, b, c)$, et par conséquent d'obtenir $\tilde{\phi}_{i}=a$. Comme l'erreur quadratique moyenne $E(a, b, c)$ (Éq. (4)) est constituée de la somme des erreurs sur les développements limités aux $\lambda$ points maîtres, elle peut être considérée comme une indication de l'erreur locale d'interpolation, $\Delta \tilde{\phi}_{i}$, de $\tilde{\phi}_{i}$ au point $i$. Pour être homogène à $\tilde{\phi}_{i}$, cette indication d'erreur $\Delta \tilde{\phi}_{i}$ est plus précisément calculée à partir de $E(a, b, c)$ comme suit :

$$
\Delta \tilde{\phi}_{i}=\sqrt{2\left(\sum_{j=1, \ldots, \lambda} \omega_{j}\right)^{-1} E(a, b, c)}
$$

\section{Couplage de NSGA-II au métamodèle (M-NSGA-II)}

Dans cette section, nous considérons trois manières de coupler ce métamodèle à l'algorithme NSGA-II; elles diffèrent par le choix des points maîtres au cours des itérations.

\subsection{Métamodèle constant : M-Constant}

Comme cela est souvent le cas avec les métamodèles utilisant la méthodologie des surfaces de réponse $[10,14,17]$, le métamodèle constant M-Constant consiste tout simplement à utiliser un nombre fixe de points maîtres. Une fois construit, il est utilisé en remplacement des évaluations exactes durant toutes les itérations de l'AEMO. Cela revient à utiliser l'AEMO pour calculer le front de Pareto du métamodèle. Ce dernier est construit à partir des $\lambda=\lambda_{\max }(=20 n)$ premiers individus de la population initiale $P_{0}$, générée aléatoirement par NSGA-II, et de taille $N=50 n$. Il est noté $M_{0}$. Autrement dit, les $20 n$ premiers individus de la population initiale $P_{0}$ sont évalués exactement alors que le reste des individus de cette population, et de toutes les générations suivantes $Q_{t}$, sont approximés par $M_{0}$.

\subsection{Métamodèle actualisé aléatoirement : M-Actualisé}

Comme cela a été constaté dans la littérature [14], et comme nous le verrons par la suite, le métamodèle constant est mal adapté aux fonctions complexes ayant de fortes variations locales. Un premier type d'amélioration est apporté en enrichissant le métamodèle avec de nouveaux points maîtres localisés au voisinage de l'optimum, c'est-à-dire du front de Pareto pour un POMO. Nous le nommons M-Actualisé. Le métamodèle initial, $M_{0}$, est construit comme précédemment mais avec seulement un nombre limité de points maîtres, correspondant au nombre minimal de points nécessaire pour avoir une interpolation quadratique, c'est-à-dire pour que la matrice du système linéaire (Éq. (5)) soit inversible. Nous retenons 
donc les $\lambda=1+n+n \frac{n+1}{2}$ premiers individus de la population initiale $P_{0}$, sous la contrainte que $\lambda \leq \lambda_{\max }$. La population $P_{0}$ étant générée aléatoirement, cela revient, comme précédemment, à sélectionner aléatoirement $\lambda$ points dans l'espace paramétrique. Les fonctions coût sont alors évaluées exactement en ces points. Le métamodèle $M_{0}$ est ensuite utilisé pour approximer les valeurs des autres individus de $P_{0}$. À chaque nouvelle génération $t$ de l'algorithme génétique, et tant que $t \leq N_{u}, N_{u}$ étant le nombre maximal de générations où l'enrichissement est autorisé (Éq. (9)), le métamodèle $M_{t}$ est amélioré en lui ajoutant $\theta=2^{M-1}$ nouveaux points maîtres, où les fonctions coût sont évaluées exactement pour construire $M_{t+1}$. Ce nouveau métamodèle est alors utilisé pour évaluer les valeurs des fonctions pour tous les individus de la population enfant $Q_{t}$, ainsi que pour corriger les évaluations précédentes de la population parent $P_{t}$.

$$
N_{u}=\frac{\lambda_{\max }-\lambda}{\theta}
$$

Les $\theta$ premiers individus de la population enfant $Q_{t}$, sont retenus, dans l'ordre résultant de leur génération par les opérateurs génétiques. Ainsi, à chaque génération, le métamodèle s'enrichit de nouveaux points maîtres, potentiellement plus proches du front de Pareto, dans la mesure où l'AEMO converge vers celui-ci.

\subsection{Métamodèle évolutif avec fonction mérite : M-Évolutif}

Le métamodèle évolution M-Évolutif diffère de M-Actualisé uniquement par la manière de sélectionner les $\theta$ nouveaux points maîtres parmi la population $P_{t}$ des parents. Plutôt que de les choisir de manière quasialéatoire, les individus sont classés suivant le critère de dominance et de distance d'encombrement, en fonction de leur position croissante sur les différents fronts et de leur distance décroissante sur chaque front. Cela permet de sélectionner les points les plus intéressants de $P_{t}$, au sens du métamodèle $M_{t}$, et donc d'améliorer $M_{t}$ dans cette partie de l'espace. Toutefois, $M_{t}$ est seulement une approximation du modèle réel. Il est donc possible que l'amélioration obtenue à partir de ces points ne soit pas aussi intéressante qu'attendu, alors que des points non retenus auraient pu s'avérer bien plus intéressants. Cependant, ayant évalué une indication de cette erreur d'approximation locale (Éq. (8)), nous pouvons estimer la fiabilité des prédictions et orienter notre choix vers les points potentiellement les plus intéressants. Pour cela, nous utilisons la fonction mérite, $\tilde{\phi}_{m}=\tilde{\phi}-\Delta \tilde{\phi}$, déjà utilisée par de nombreux auteurs [11-14], à la place de $\tilde{\phi}$ lors du calcul des critères de dominance et d'encombrement. $\tilde{\phi}_{m}$ représente en effet la meilleure valeur que $\phi$ puisse atteindre compte tenu de l'erreur d'approximation. Les valeurs minimales de $\tilde{\phi}_{m}$ correspondent à des valeurs faibles de $\tilde{\phi}$ ou à des valeurs fortes de $\Delta \tilde{\phi}$, c'est-à-dire à des zones inexplorées de l'espace paramétrique. M-Évolutif permet ainsi d'associer une meilleure exploration de l'espace paramétrique et une exploitation des zones où le front de Pareto semble être localisé.

\section{Problèmes analytiques}

Les problèmes analytiques sont fréquemment utilisés pour tester, comparer et évaluer la performance de nouveaux AEMOs. Ils ont pour propriétés d'être simples à utiliser et de permettre le calcul analytique du front de Pareto [18-20]. Nous comparons les résultats obtenus avec les différentes méthodes de couplage du métamodèle, M-Constant, M-Actualisé et M-Évolutif, à NSGA-II standard (AEMO sans métamodèle) sur plusieurs de ces problèmes, et présentons les résultats les plus caractéristiques.

\subsection{Min-Ex}

Le problème Min-Ex (Éq. (10)) porte sur deux fonctions coût et deux variables. Il est proposé par Deb [1], et a comme solution un front optimal de Pareto convexe. Bien que ce problème ait l'air simple, les fonctions coût sont conflictuelles ce qui conduit à un ensemble de solutions optimales. Le problème (10) revient à minimiser $\frac{1}{x_{1}}$ puisque la minimisation de $f_{2}\left(x_{1}, x_{2}\right)$ impose $x_{2}=0$. Le front analytique de Pareto s'écrit donc : $f_{2}=\frac{1}{f_{1}}$.

$$
\text { Min-Ex : }\left\{\begin{array}{l}
\text { Minimiser } f_{1}\left(x_{1}, x_{2}\right)=x_{1} \\
\text { Minimiser } f_{2}\left(x_{1}, x_{2}\right)=\frac{1+x_{2}}{x_{1}} \\
0,1 \leq x_{1} \leq 1 \text { et } 0 \leq x_{2} \leq 5
\end{array}\right.
$$

La figure 2 montre que les différentes approches permettent toutes d'assez bien retrouver le front optimal de Pareto. Certaines sont aussi précises que NSGA-II standard (superposé au front analytique), qui requiert pourtant 140 fois plus de calculs exacts (5500 dans ce cas). Nous y voyons aussi l'importance d'actualiser le métamodèle, car avec seulement 40 calculs exacts, M-Constant ne permet pas d'obtenir une approximation satisfaisante du front optimal de Pareto sur toute sa longueur. Cela vient du fait que le métamodèle initial n'est pas assez précis au voisinage du front, correspondant à $x_{2}=0$. En revanche, pour les deux métamodèles évolutifs, M-Actualisé et M-Évolutif, on obtient un front tout aussi précis qu'avec NSGA-II standard. La figure 3 montre les deux fonctions approchées calculées et utilisées par M-Évolutif sur l'ensemble de l'espace paramétrique, en comparaison avec les surfaces analytiques. Nous observons que si $f_{1}$, très simple, est parfaitement approchée, $f_{2}$ est parfaitement approchée au voisinage du front de Pareto $\left(x_{2}=0\right)$ et avec moins de précision plus loin.

\section{$6.2 \mathrm{SCH} 2$}

Le second problème (Éq. (11)), proposé par Schaffer, est également très souvent utilisé pour tester la robustesse des AEMOs. Il concerne la minimisation de deux fonctions coût avec une seule variable. L'ensemble 


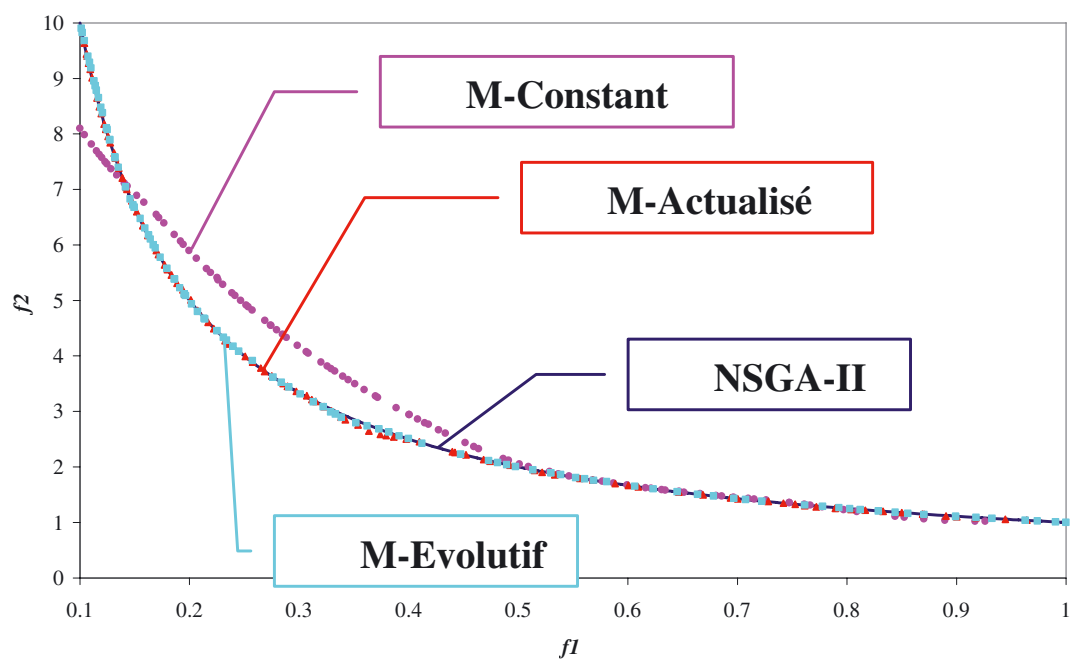

Fig. 2. Comparaison des fronts optimaux de Pareto obtenus pour le POMO Min-Ex avec NSGA-II, M-Constant, M-Actualisé et M-Évolutif.
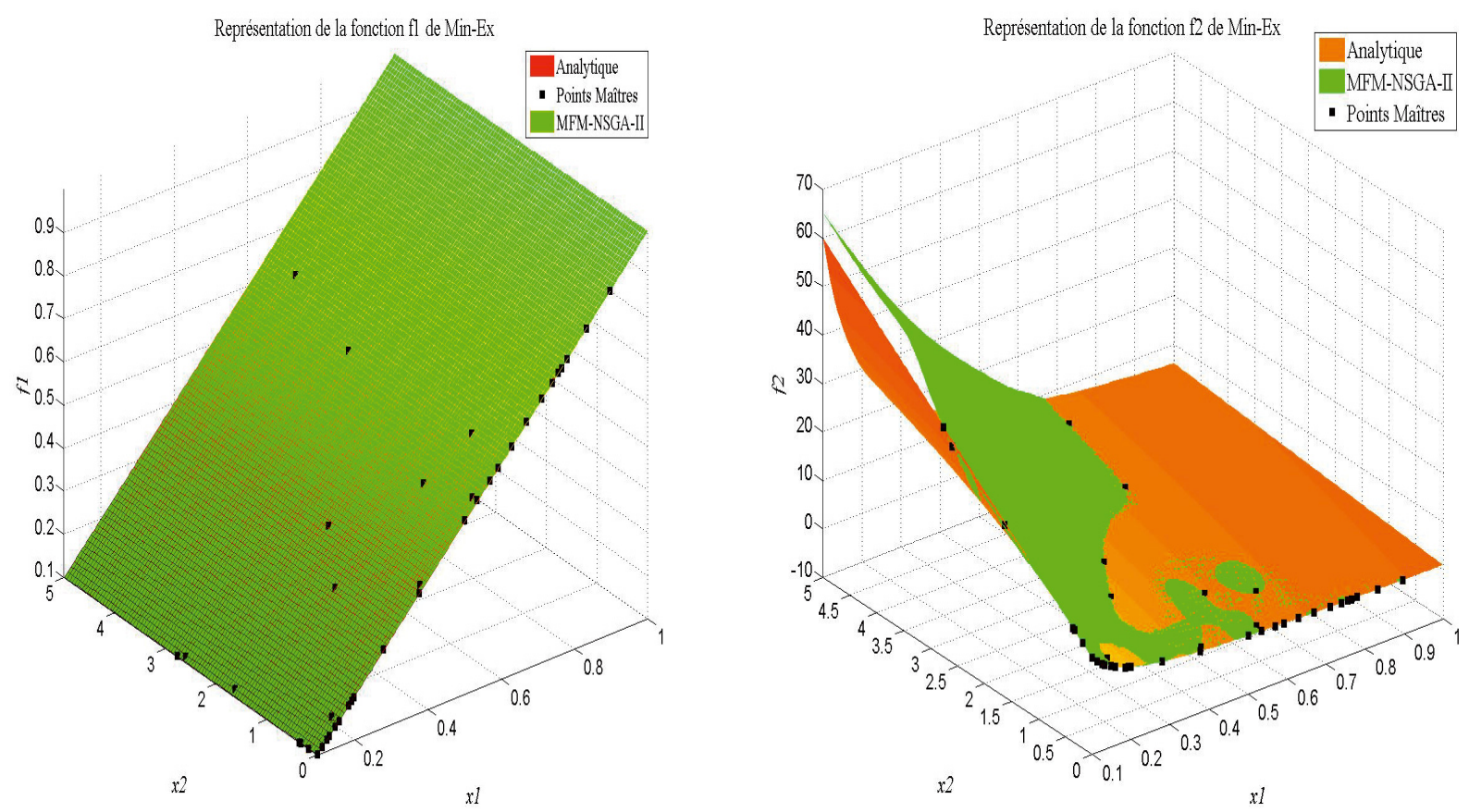

Fig. 3. Représentation des fonctions $f_{1}$ (gauche) et $f_{2}$ (droite) de Min-Ex obtenues par M-Évolutif (surfaces vertes), sur la base des points maîtres (noir) et les solutions analytiques (orange).

optimal de Pareto est formé par deux fronts discontinus : $x^{*} \in\{[1,2] \cup[4,5]\}$.

\section{$\mathrm{SCH} 2$ :}

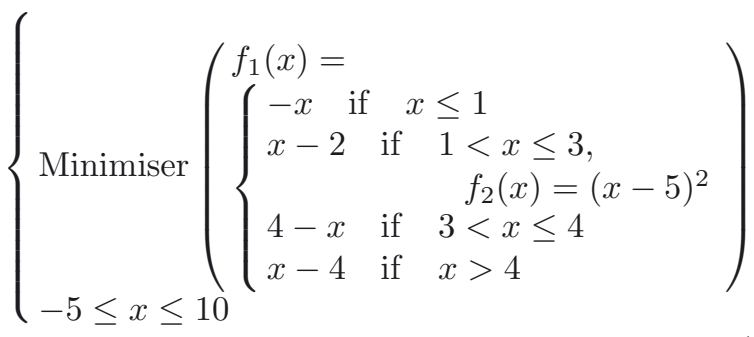

Tableau 1. Paramétrages des quatre algorithmes.

\begin{tabular}{cccccc}
\hline Algorithmes & $N$ & $N_{u}$ & $\lambda$ & $\lambda_{\max }$ & $\theta$ \\
\hline NSGA-II & 100 & 50 & - & - & - \\
M-Constant & 50 & - & 20 & 20 & - \\
M-Actualisé & 50 & 8 & 3 & 20 & 2 \\
M-Évolutif & 50 & 8 & 3 & 20 & 2 \\
\hline
\end{tabular}

Le tableau 1 présente les paramétrages utilisés avec les quatre algorithmes. De nouveau (voir Fig. 4), MConstant permet d'obtenir l'allure des deux branches du front optimal de Pareto, mais ne parvient pas à correctement estimer sa branche de gauche, qui sont par ailleurs 


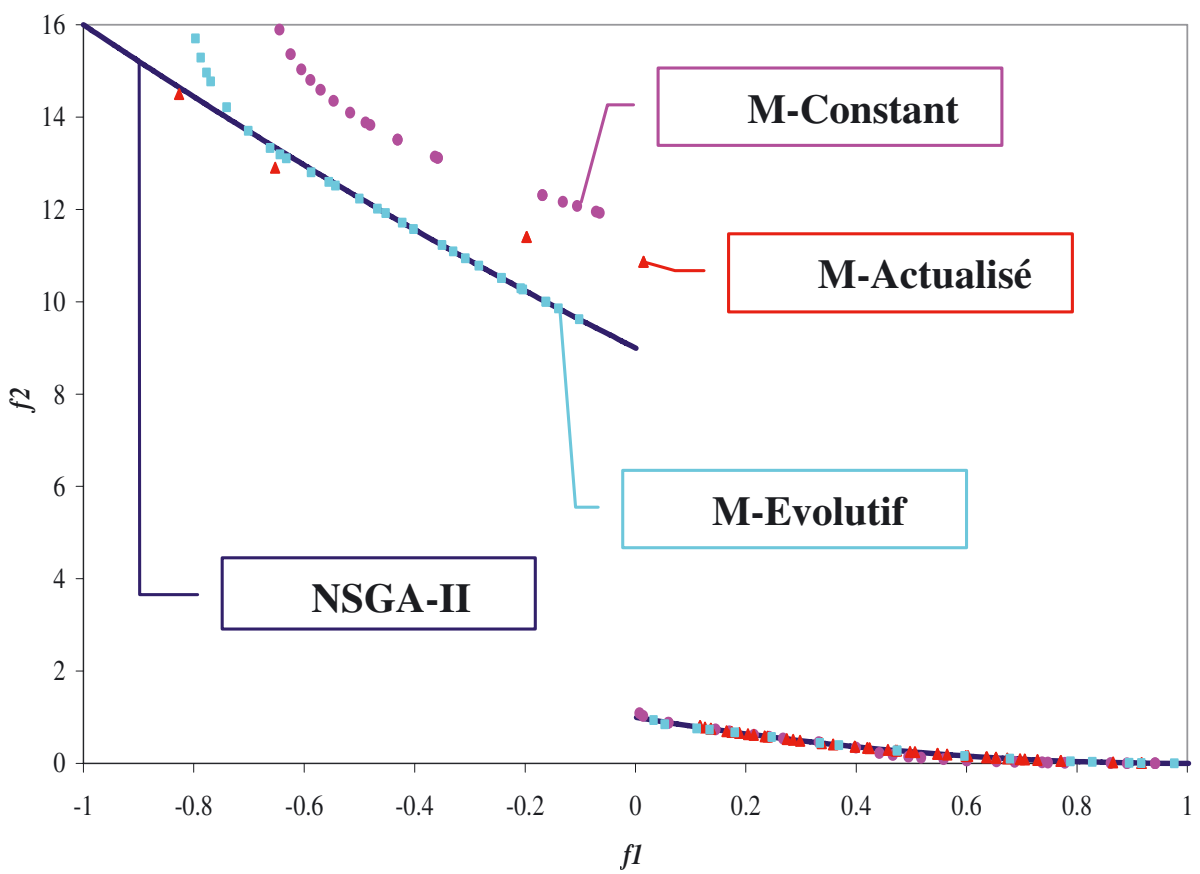

Fig. 4. Comparaison entre les fronts optimaux de Pareto obtenus pour le POMO SCH2 avec NSGA-II, M-Constant, M-Actualisé et M-Évolutif.

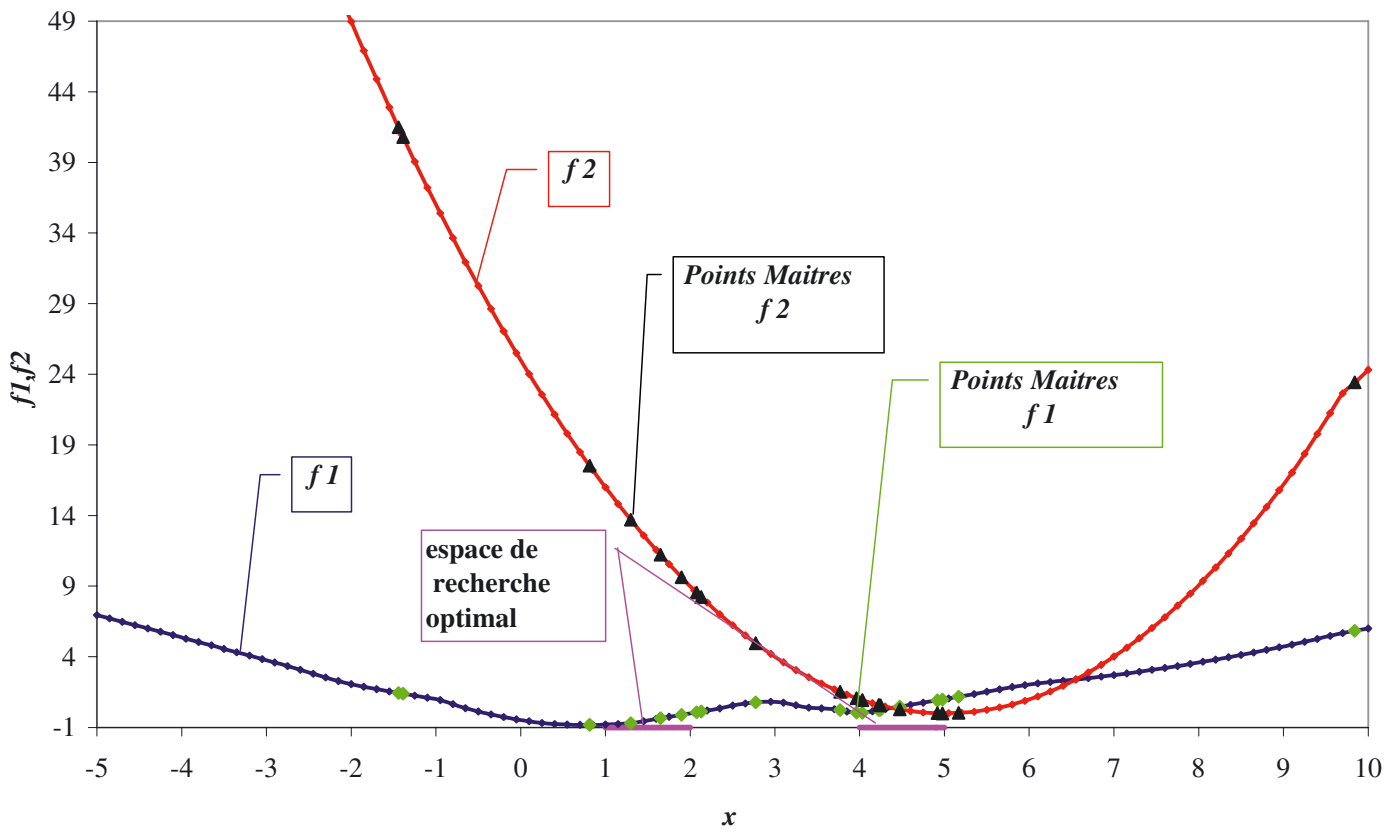

Fig. 5. Représentation des fonctions $f_{1}$ et $f_{2}$ de SCH2 obtenus par M-Évolutif.

très précisément calculées en 5500 calculs exacts avec NSGA-II. Avec davantage de calculs exacts, 100 au lieu de 20, M-Constant parvient à obtenir une bonne approximation des deux branches du front. En restant limité à 20 calculs, M-Actualisé offre une solution un peu plus précise que M-Constant, mais a également du mal à approcher la branche de gauche. En revanche, M-Évolutif donne des résultats excellents et offre une approximation semblable à NSGA-II en 250 fois moins de calculs. La figure 5 montre que ce métamodèle, bien qu'il soit initialisé avec un petit nombre (3 dans ce cas) de points maîtres répartis aléatoirement, est capable de générer de nouveaux points au voisinage des solutions optimales du POMO, ce qui permet une bonne approximation des fonctions et la convergence de l'algorithme.

\subsection{KUR}

KUR (Éq. (12)) est un problème d'optimisation multiobjectifs à deux fonctions coût et trois variables de 


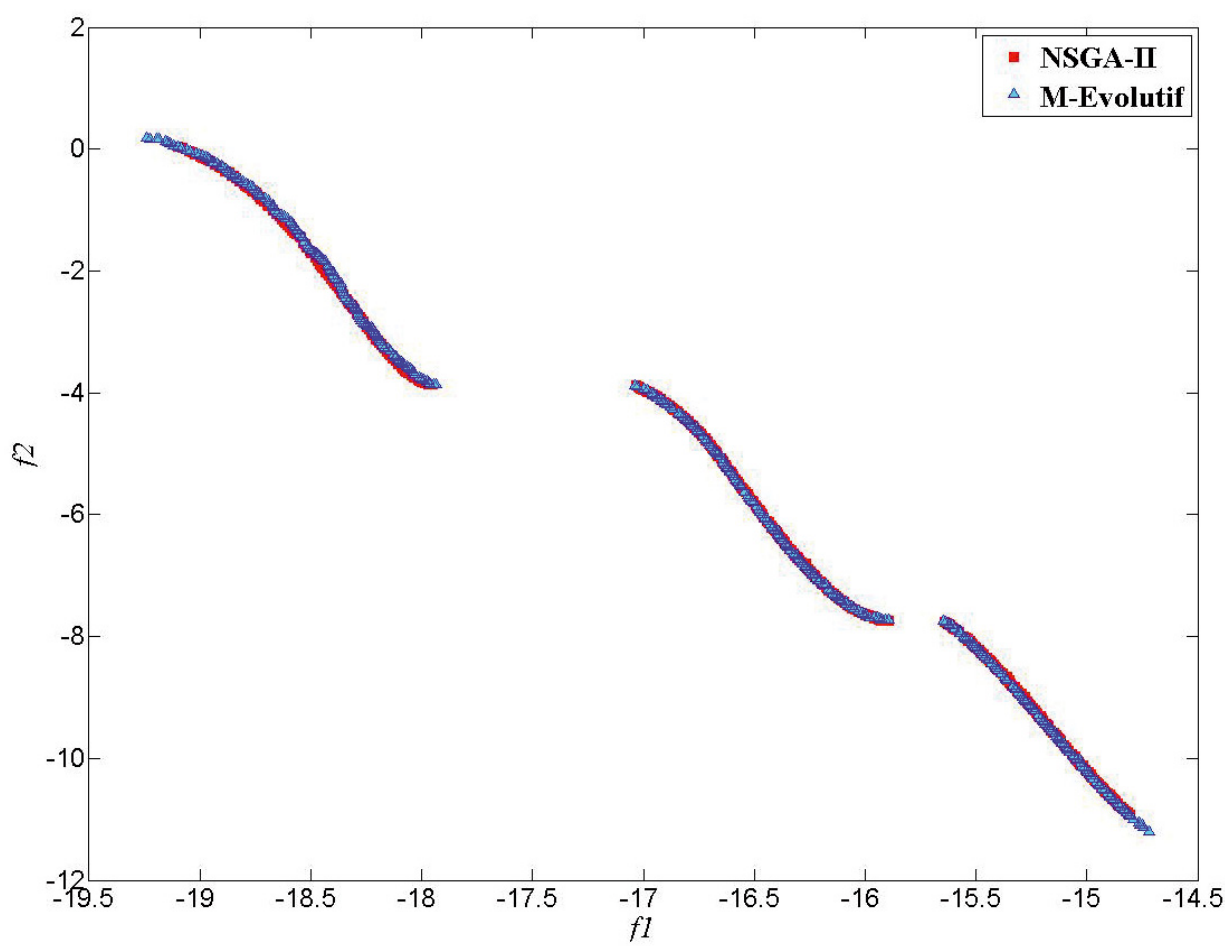

Fig. 6. Comparaison entre les fronts optimaux de Pareto obtenus pour le POMO KUR avec NSGA-II (rouge) et M-Évolutif (bleu).

décisions. Son front optimal de Pareto est non convexe et discontinu, constitué de trois branches.

$$
\mathrm{KUR}:\left\{\begin{array}{rr}
\text { Minimiser } \quad f_{1}(X)=\sum_{i=1}^{2}[-10 \exp (-0,2 \\
& \left.\left.\times \sqrt{x_{i}^{2}+x_{i+1}^{2}}\right)\right] \\
\text { Minimiser } \quad f_{2}(X)= & \sum_{i=1}^{3}\left[\left|x_{i}\right|^{0,8}+5 \sin \left(x_{i}^{3}\right)\right] \\
& -5 \leq x_{i} \leq 5, \quad i=1,2,3
\end{array}\right.
$$

Sur ce problème NSGA-II rencontre des difficultés à converger, aussi le nombre de calculs exacts est-il poussé à 40400 pour parvenir à trouver la solution du problème (voir Fig. 6). Avec les algorithmes proposés, il est également nécessaire d'augmenter le nombre de calculs exacts, mais dans les mêmes proportions, aussi utilisera-ton 600 points maîtres. M-Constant et M-Actualisé ne permettent pas de trouver le front de Pareto. En revanche, M-Évolutif trouve le même front optimal que NSGA-II (voir Fig. 6) avec trois branches et un gain de 98,5\% en nombre d'évaluations. La figure 7 montre la complexité de la localisation des solutions dans l'espace paramétrique, avec de fortes discontinuités.

\subsection{Min-Ex avec Contraintes}

Ce problème (Éq. (13)) proposé par Deb [1] est semblable à Min-Ex (10) avec deux fonctions coût et deux variables, mais on lui a ajouté deux contraintes implicites d'inégalité. Seul M-Évolutif est étudié. La figure 8 montre sa capacité à prendre en compte les contraintes, et à trouver de nouveau le front de Pareto en seulement 40 calculs exacts.

$$
\text { Min-Ex : }\left\{\begin{array}{l}
\text { Minimiser }\left(f_{1}\left(x_{1}, x_{2}\right)=x_{1},\right. \\
\left.f_{2}\left(x_{1}, x_{2}\right)=\frac{1+x_{2}}{x_{1}}\right) \\
g_{1}\left(x_{1}, x_{2}\right)=x_{2}+9 x_{1} \geq 6 ; \\
g_{2}\left(x_{1}, x_{2}\right)=-x_{2}+9 x_{1} \geq 1 \\
0,1 \leq x_{1} \leq 1 \\
0 \leq x_{2} \leq 5
\end{array}\right.
$$

\section{Problème de mise en forme}

Dans cette partie, M-Évolutif est utilisé pour résoudre deux problèmes d'optimisation en mise en forme des matériaux. Le premier est mono-objectif et le deuxième est multi-objectifs.

\subsection{Forgeage d'une bielle}

Nous considérons l'optimisation de la forme d'un lopin pour le forgeage d'une bielle. Ce lopin a une forme cylindrique (voir Fig. 9) définie en plusieurs parties permettant d'assurer une bonne répartition de la matière 


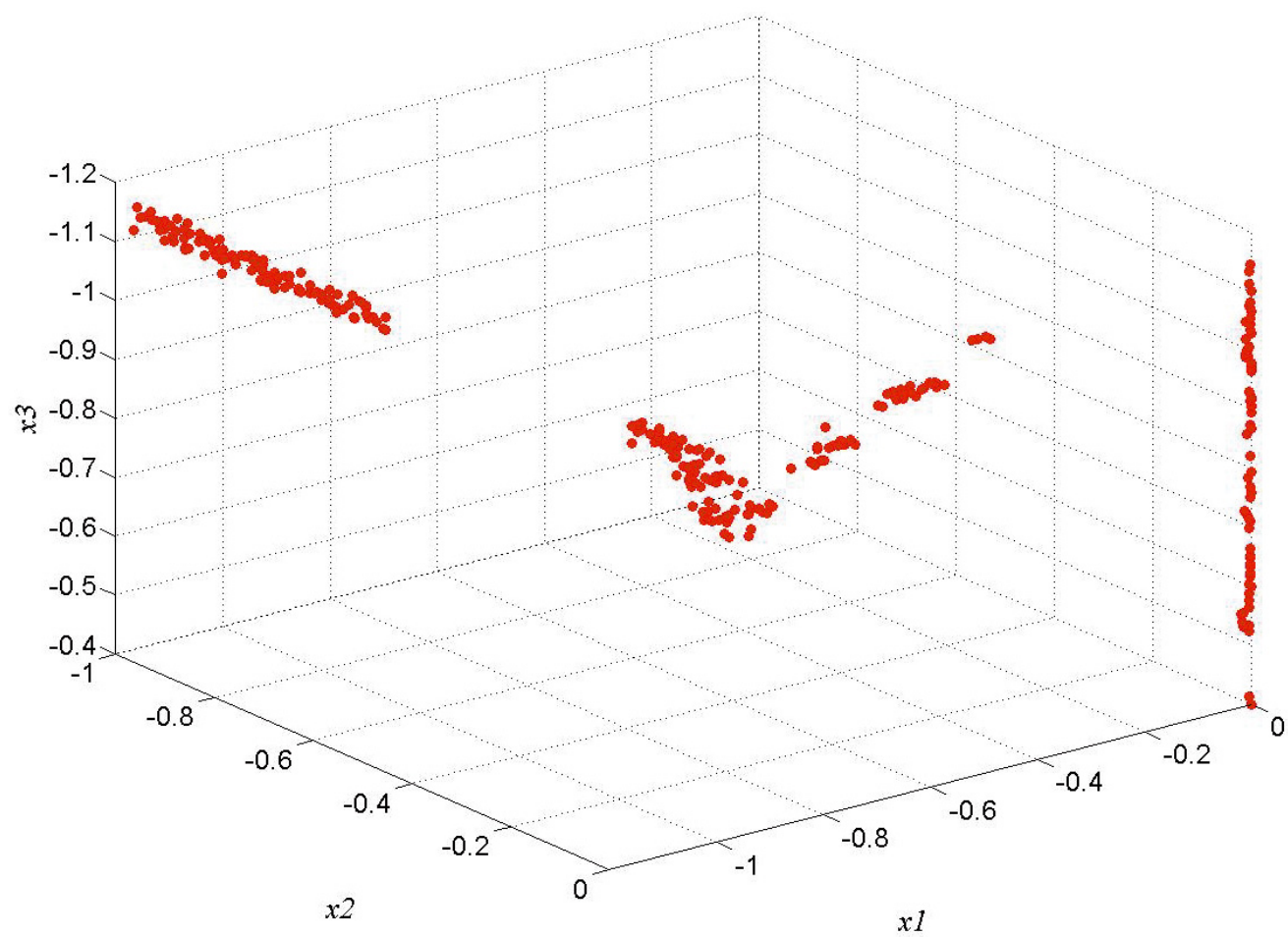

Fig. 7. Répartition des solutions optimales obtenues par M-Évolutif dans l'espace de recherche.

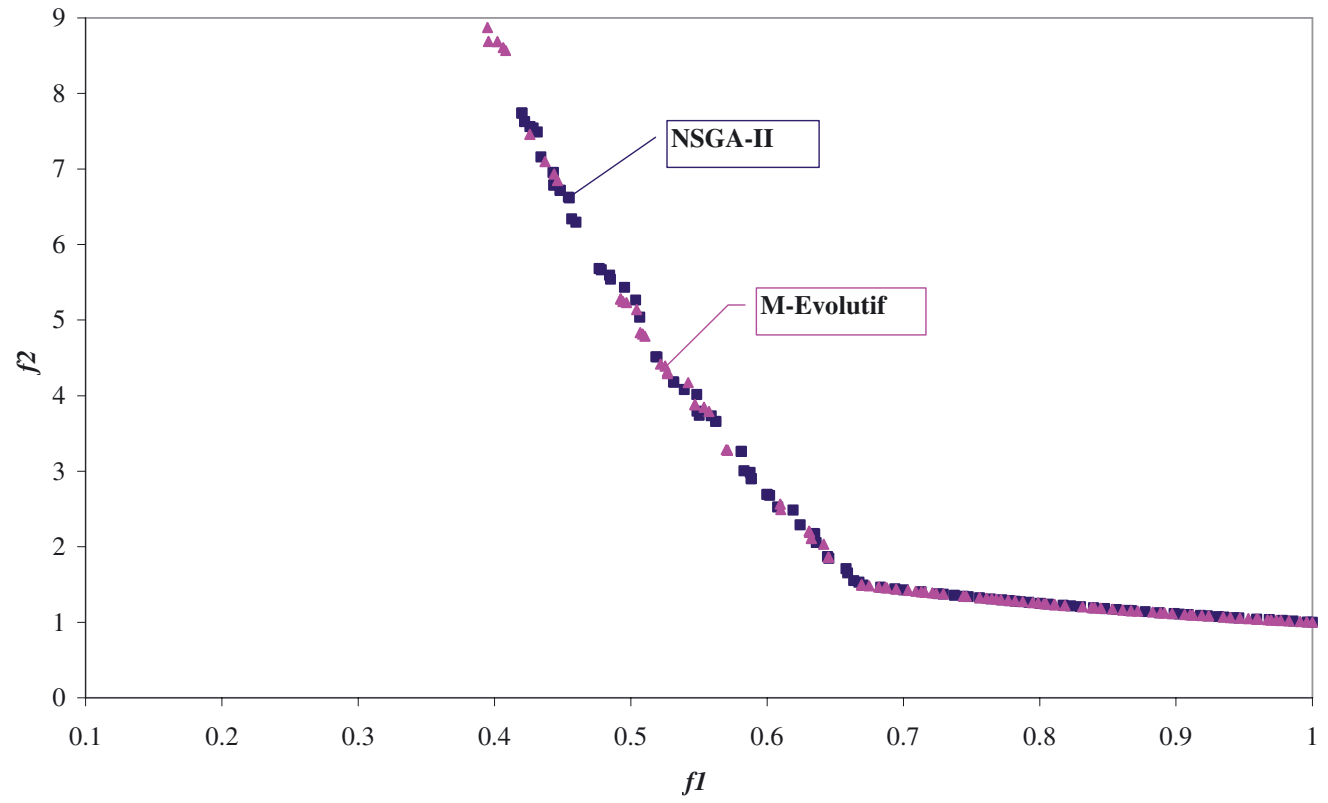

Fig. 8. Comparaison entre les fronts optimaux de Pareto obtenus pour le POMO Min-Ex avec contraintes avec NSGA-II et M-Évolutif.

pour le forgeage de la bielle. Nous cherchons à minimiser le volume de matière, la fonction $f_{1}$ du problème (Éq. (14)), en effectuant une homothétie de la géométrie du cylindre à partir de son axe. Le paramètre d'optimisation est donc ce rapport d'homothétie vis-à-vis de la géométrie initiale. La contrainte d'optimisation, la fonction $f_{2}$ du problème (Éq. (14)), est d'obtenir une bielle en fin de forgeage, c'est-à-dire de remplir les parties actives des matrices (voir Fig. 10) - la partie des matrices correspondant à la bavure n'est donc pas prise en compte. Ce problème a été résolu par l'algorithme monoobjectif SE-META, proposé par Emmerich [9], en traitant la contrainte par pénalisation et en additionnant les deux fonctions coût (14). Il est traité de la même manière 


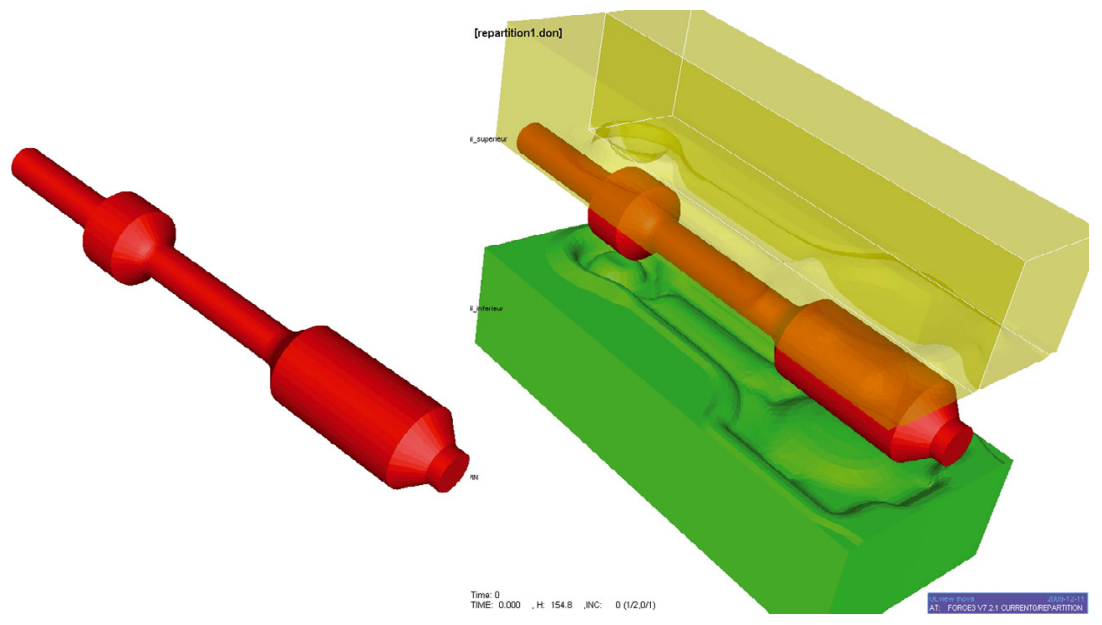

Fig. 9. Préforme initiale, outil supérieur (en haut), lopin (au milieu) et outil inférieur (en bas).

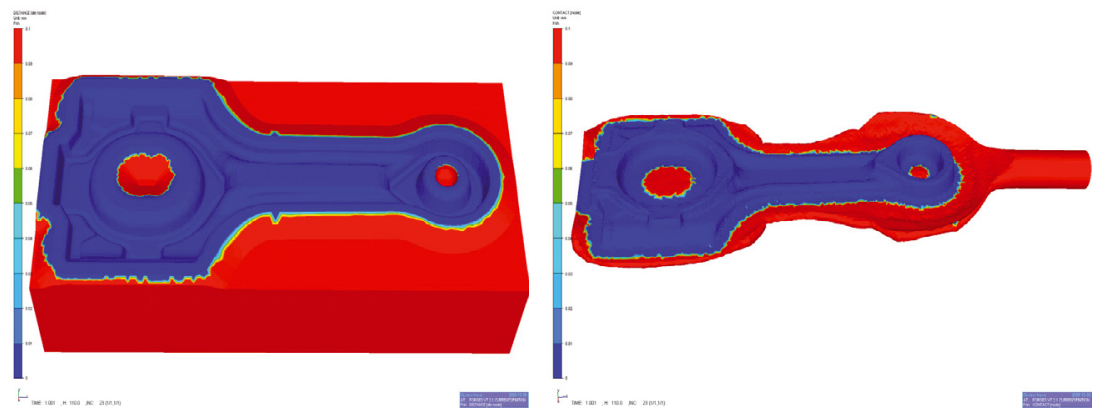

Fig. 10. Forgeage d'une bielle avec Forge $3^{\circledR}$ : iso valeurs du contact sur la pièce (droite) et sur l'outil (gauche) - géométrie de la pièce obtenue après forgeage du lopin (droite).

Tableau 2. Comparaison entre M-Évolutif et SE-META pour le forgeage d'une bielle.

\begin{tabular}{ccc}
\hline Algorithmes & $x$ & $\min f_{(x)}$ \\
\hline SE-META & 1,021 & 1,099 \\
M-Évolutif & 1,015 & 1,086 \\
\hline
\end{tabular}

avec M-Évolutif, en 20 calculs exacts, tout comme pour SE-META.

Forgeage d'une bielle : $\left\{\begin{array}{r}\text { Minimiser } f(x)=\left(f_{1}(x)+f_{2}(x)\right) \\ 0,8 \leq x \leq 1,05 \\ \text { Avec } f_{1}(x)=\text { volume et } \\ f_{2}(x)=\text { non-remplissage }\end{array}\right.$

Les résultats du tableau 2 montrent que M-Évolutif trouve une solution comparable à SE-META, mais correspondant à un volume plus faible et une valeur de fonction coût légèrement inférieure.

\subsection{Tréfilage d'un fil}

Nous considérons ici le problème d'optimisation de la géométrie (voir Fig. 11) d'une filière utilisée pour le tréfilage de fil (voir maillage du fil Fig. 12). Ces filières sont généralement dimensionnées (demi-angle d'entrée $\alpha$, longueur de portée $L$, angle de sortie, voir Fig. 11) pour minimiser l'effort maximal d'étirage, $F_{\max }$. Toutefois, des ruptures de fil étant parfois observées, nous intéressons à minimiser l'endommagement maximal $D_{\max }$ du matériau durant le procédé, calculé ici par un critère de Latham et Cockcroft :

$$
D_{\max }=\int_{0}^{\varepsilon_{f}} \max \left(0, \sigma_{1}\right) \mathrm{d} \varepsilon_{\mathrm{p}}
$$

Nous obtenons ainsi un POMO (Éq. (5)) à deux fonctions coût, $F_{\max }$ et $D_{\max }$, et à deux paramètres, la longueur de la portée $L$ et le demi-angle $\alpha$ (voir Fig. 11) que nous résolvons avec M-Évolutif en 40 simulations exactes de tréfilage.

$$
\text { Tréfilage }\left\{\begin{array}{cc}
\text { Minimiser } & f_{1}\left(x_{1}, x_{2}\right)=D_{\max } \\
\text { Minimiser } & f_{2}\left(x_{1}, x_{2}\right)=F_{\max } \\
& 0,1 \leq x_{1}, x_{2} \leq 2
\end{array}\right.
$$

La figure 13 présente le front de Pareto obtenu, avec une excellente répartition des points sur l'ensemble de la courbe. Nous observons que les deux fonctions coût sont antagonistes. L'analyse des résultats montre tout d'abord que les deux fonctions coût sont principalement sensibles 


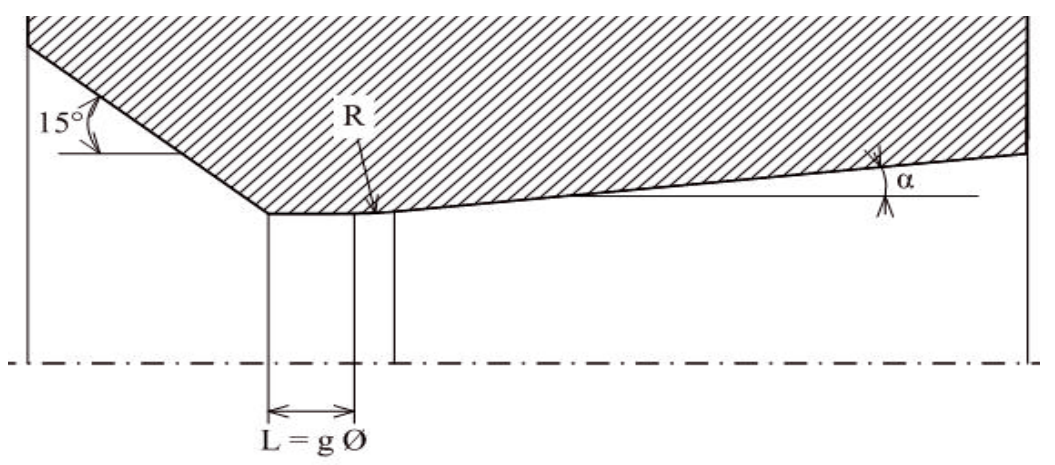

Fig. 11. Filière de tréfilage avec ses paramètres d'optimisation.

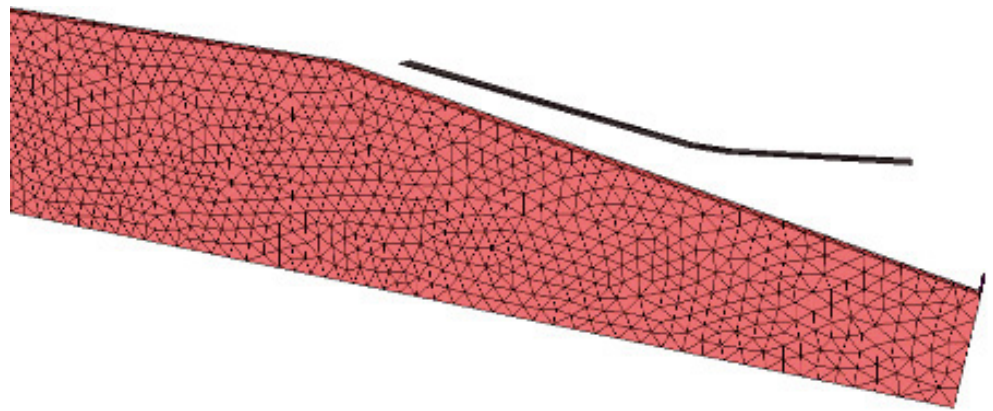

Fig. 12. Simulation du tréfilage d'un fil - maillage de calcul.

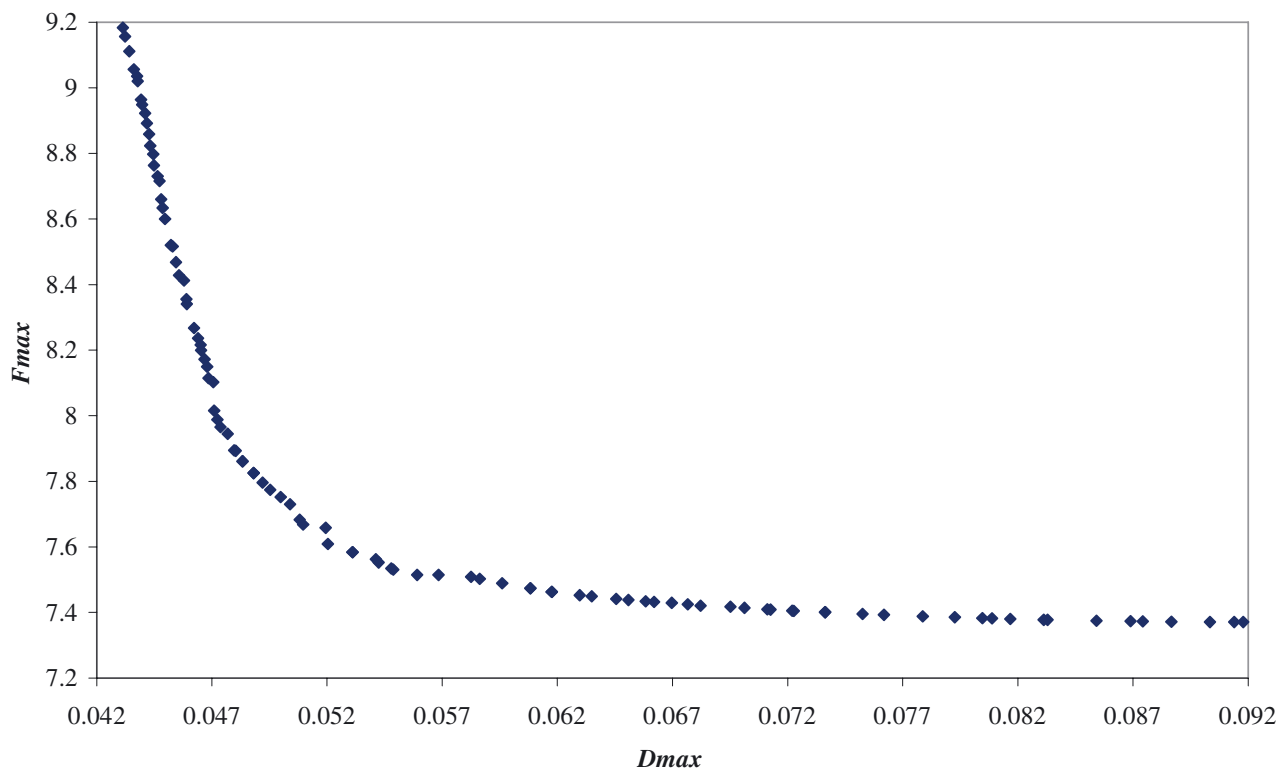

Fig. 13. Front optimal de Pareto du POMO tréfilage obtenu par M-Évolutif.

à l'angle de filage; la longueur de portée influence peu les résultats. Par ailleurs, la théorie du filage indique qu'il existe un angle de filière optimal pour minimiser $F_{\max }$, et que cet angle dépend du coefficient de frottement. En revanche, les calculs montrent qu'il n'existe pas d'angle minimal pour l'endommagement. Plus l'angle est faible, plus $D_{\max }$ est faible. De ce point de vue, la solution optimale est donc l'angle minimal de filière qu'il est possible de réaliser industriellement.
En une seule optimisation, M-Évolutif permet de calculer l'ensemble des compromis possibles (Fig. 13). Ainsi, plutôt que de retenir la solution à l'extrême droite de la courbe, qui correspond à la valeur optimale de l'angle de filage vis-à-vis de $F_{\max }$ mais qui se traduit par une valeur élevée de l'endommagement $D_{\max }$, nous pouvons préférer une solution située dans le premier tiers gauche de la courbe : en acceptant ainsi une légère augmentation de l'effort maximal $F_{\max }$ (de quelques dizaines de pourcents), 
nous pouvons réduire très significativement l'endommagement (presque de moitié).

\section{Conclusion}

À l'issue de travaux précédents [16], nous avions conclu que le couplage d'algorithmes génétiques à un métamodèle permettait de résoudre des problèmes d'optimisation mono-objectif fortement non convexes contenant de nombreux extrema, à un coût de calcul limité et contrôlé. Nous constatons maintenant qu'il est possible d'étendre cette approche à une problématique multiobjectifs, qui nécessite cette fois la recherche d'un ensemble de solutions optimales, le front de Pareto, pour un coût en nombre de calculs exacts à peine supérieur, et limité à une centaine. Sur les fonctions analytiques étudiées, continues et discontinues, nous observons qu'il est très important de mettre à jour le métamodèle aux cours des itérations d'optimisation, ce qui permet d'affiner la précision de ce dernier autour du front de Pareto. L'algorithme M-Évolutif, basé sur une actualisation à partir des meilleurs parents sélectionnés par la fonction mérite, permet ainsi d'obtenir des résultats très satisfaisants sur tous les cas étudiés.

L'application au premier problème de mise en forme des métaux montre que cet algorithme multi-objectif est tout aussi performant qu'un algorithme mono-objectif de référence, pour un problème à une seule fonction coût. La seconde application de tréfilage illustre la possibilité d'obtenir, en une seule opération d'optimisation, des informations sur le procédé beaucoup plus complexes et intéressantes. Elles permettent ici de suggérer de nouvelles solutions optimales, différentes de celles qui émergent de l'optimisation mono-objectif habituellement utilisée, et de sélectionner le meilleur compromis en visualisant l'effet des antagonismes sur le front de Pareto.

Remerciements. Ce projet a été réalisé dans le cadre du projet ANR LOGIC. Les auteurs remercient chaleureusement Stéphane Marie de la société Transvalor, Thomas Massé et Pierre Montmitonnet du CEMEF, pour leur contribution à la simulation des deux derniers cas d'optimisation.

\section{Références}

[1] K. Deb, Multi-Objective Optimisation using Evolutionary Algorithms, John Wiley \& Sons, Chichester, 2001

[2] M. Emmerich, N. Beume, B. Naujoks, An EMO algorithm using the hyper volume measure as selection criterion, Evolutionary Multi-Criterion Optimization 3410 (2005) $62-76$

[3] N. Beume, B. Naujoks, M. Emmerich, SMS-EMOA: Multi-objective selection based on dominated hypervolume, Eur. J. Res. 181 (2007) 1653-1669

[4] J. Krok, An Extended Approach to Error Control in Experimental and Numerical Data Smoothing and Evaluation Using the Meshless FDM, Meshfree Computational Mechanics 11 (2002) 913-945
[5] K. Pawan, S. Nain, K. Deb, A Multi-Objective Optimization Procedure with Successive Approximate Models, KanGAL Report Number 2005002

[6] K. Pawan, S. Nain, K. Deb, A Multi-Objective Search and Optimization Procedure with Successive Approximate Models, KanGAL Report Number 2004012

[7] T. Goal, R. Vaidyanathan, R.T. Haftka, W. Shyy, N.V. Queipo, K. Tucker, Response surface approximation of Pareto optimal front in multi-objective optimization, Comput. Methods Appl. Mech. Eng. 196 (2007) 879-893

[8] K. Deb, A. Pratap, S. Agarwal, T. Meyarivan, A Fast and Elitist Multi-Objective Genetic Algorithm: NSGAII, KanGAL Report Number 200001

[9] M. Emmerich, B. Naujoks, Metamodel-Assisted Multiobjective Optimization with Implicit Constraints and its Application in Airfoil Design, International Conference and Advanced Course ERCOFTAC, Athens, Greece, 2004

[10] G. Ingarao, R.D. Lorenzo, F. Micari. Internal pressure and counter-punch action design in Y-shaped tube hydroforming processes: A multi-objective optimisation approach, Comput. Struct. 87 (2009) 591-602

[11] M. Emmerich, A. Giotis, M. Özdemir, T. Bäck, K. Giannakoglou, Metamodel-assisted evolution strategies,International Conference on parallel problem solving from nature Springer, Berlin, Germany, 2002

[12] M. Emmerich, K. Giannakoglou, B. Naujoks, Single- and Multiobjective Evolutionary Optimization Assisted by Gaussian Random Field Metamodels, IEEE Trans. Evol. Comput. 10 (2006) 421-439

[13] M. Emmerich, B. Naujoks, Metamodel-Assisted Multiobjective Optimization Algorithms and their application in Airfoil Design, in Proc. 5th Int. Conf. Adaptive Computing in Design and Manufacture VI (ACDM), Springer, London, 2004, pp. 249-260

[14] M.H.A Bonte, L. Fourment, T.T. Do, A.H. Van den Boogaard, J. Huétink. Optimisation of metal forming processes using Finite Element simulations: A sequential approximate optimization algorithm and its comparision to other algorithms by application to forging, Structural and Multidisciplinary optimization

[15] T. Liszka, J. Orkisz, The finite difference method at arbitrary irregular grids and its application in applied mechanics, Comput. Struct. 11 (1980) 83-95

[16] M.T.T. Do, Optimisation de forme en forgeage 3D, Ph.D. thesis, Mines ParisTech.

[17] P. Breitkopf, H. Naceur, A. Rassineux, P. Villon. Moving Least squares response surface approximation: Formulation and metal forming applications, Comput. Struct. 83 (2005) 1411-1428

[18] K. Deb, L. Thiele, M. Laumanns, E. Zitzler, Scalable Test Problems for Evolutionary Multi-Objective Optimization, in A. Abraham, L. Jain, R. Goldberg (éds.), Evolutionary Multiobjective Optimization. Theoretical Advances and Applications, Springer, USA, 2005, pp. $105-145$

[19] K. Deb, L. Thiele, M. Laumanns, E. Zitzler, Scalable Multi-Objective Optimization Test Problems, in Congress on Evolutionary Computation (CEC) Piscataway, New Jersey, May 2002, Vol. 1, pp. 825-830

[20] K. Deb, Multi-objective genetic algorithms: Problem difficulties and construction of test problems, Evol. Comput. J. 7 (1999) 205-230 